\title{
Leisure Food: Derive Social and Cultural Entertainment through Physical Interaction with Food
}

\author{
Jun Wei ${ }^{\star}$ and Ryohei Nakatsu \\ Keio-NUS CUTE Center, IDM Institute, \\ NGS, National University of Singapore, Singapore \\ $\{$ weijun24, elenr\}@nus.edu.sg
}

\begin{abstract}
Food is not simply a source of nutrition, but also a symbolic medium for social bonds and entertainment. Beyond this, food is common artefact across cultures, embedded with internal cultural value and identity. This paper presents the notion of "Leisure Food", which explores the roles of mundane food in deriving social and cultural entertainment from people's interaction with physical food. We focus on the application of culturally based food experiences as a gateway for enriched and profound entertainment, through maximizing the physical, social and cultural affordances of food using technologies. From this notion, we developed two prototypes based on the positive interactions that people have as they eat and prepare food in their everyday lives and also extend them to remote situations. The preliminary user study demonstrates that these two prototypes can enhance the level of social engagement and culture recollection experience through the new designed forms of physical food-mediated interaction, which verified the proposed features regarding "Leisure Food".
\end{abstract}

Keywords: Food-mediated interaction, social entertainment, culture recollection, user experience.

\section{Introduction}

Entertainment, defined as occasions for creating pleasure, is so pervasive and influential in the contemporary society and adds a key dimension to the lifestyle and sense of self-satisfaction of human beings. Yet the term of entertainment is currently narrowly defined within computer-based technologies and interactive media experiences.

Essentially, entertainment is based upon providing pleasure by stimulating physical and emotional arousal 11. It is multifaceted, besides relying heavily on today's electronic devices for fun and excitement, we also need physical interaction to go beyond the barrier created by the pure digital content. One of the great

\footnotetext{
* This research is supported by the Singapore National Research Foundation under its International Research Centre @ Singapore Funding Initiative and administered by the IDM Programme Office.
} 
affordances of physical artefacts is the tangibility, which conveys emotional importance beyond the excessive information. Social and physical interactions are the new paradigms that outline a vision for the next generation of entertainment systems [2].

Beyond this, we consider culture as another important dimension for entertainment. As Stromberg indicated, "entertainment is a cultural system through which commitment to certain values is generated" 1. The culture we discuss here is not the high-level artistic culture, but the civilian culture that has been interwoven with and reflected in people's everyday life, and conveyed through the certain ways people communicate with each other and conduct their activities. Culture activities can serve as a source not only for new interaction metaphors, but further, for entertainment as well.

Therefore, after broadening our understanding of what entertainment involves in everyday experiences, we locate special concentration on the sociable, emotional and cultural aspects. The socio-cultural context is part of the user experience, as suggested by Alben's definition of user experience [3]. We believe these two features can lead to a different level of entertainment, triggering more "thick" and profound experience.

On the other hand, as we may noticed, entertainment is so woven into the fabric of current everyday life that most of the emerging devices have some playful functions inside, or are created just for entertainment, such as the smart phones, tablets, or the ambient displays for games, while the potentials of our traditional surrounding artefacts have been somewhat neglected.

In the everyday household and community life, food is often at the center of social interaction, entertainment and cultural expression. As Finkelstein proposed, "The idea of food as a source of amusement has been parallel to the experience of eating since gastronomy began" 4. One important perception of food is the enjoyment of eating and socializing at the same time. People enjoy their food, relish the practice of making it, and above all celebrate the sharing of it [5]. What's more, food is also considered as the ultimate embodiment of human culture. Special foods or significant eating events are often imbued with core social values 6 .

People are familiar with food, and food in itself encompasses a variety of characteristics that can be utilized to communicate emotional feelings, such as the food texture, shape, pattern, colour, even smell and taste. For example, the aroma of baked bread, fresh coffee, and the sensation of chocolate melting in the mouth, can often evoke a sense of comfort and contribute to the eating pleasure. Given the diversity of role that food plays in everyday life, together with its social and cultural impact, we present the notion of "Leisure Food". The core concept is to seek ways to utilize the unique potentials of food and create new channels to derive increased social and cultural entertainment from the physical food interactions.

With the pursuit of deeper emotional feeling through resonance with people's social and cultural experiences, this research looks into interesting human activities that can serve as the basis for deriving new food entertainment ideas. 
We then designed and implemented two prototypes based on the notion, and also conducted a preliminary user study to obtain users' perception and experience regarding the prototypes, so as to evaluate the effectiveness of the prototypes and also to validate the proposed notion. The contribution of this paper is its exploration into the potential space to derive entertainment in the area of human-food-human interaction, by extending and multiplexing individuals' current experiences and interactions around food.

The remaining paper is organized as follows. Section 2 introduces the social and cultural framing of entertainment and the background for "Leisure Food" paradigm, followed by the works related to food interaction for entertainment. Section 3 describes the two prototyped systems we designed for food eating and cooking respectively, and section 4 presents the results and discussion from a user study. Finally, section 5 concludes the research theories and proposes future works towards pleasurable human experience over food.

\section{Background and Related Works}

Entertainment is one of the essential elements in human society. Entertainment computing has been traditionally associated with interactive entertainment media and game playing. The rapid development of digital technology promotes the "high technologification" of various entertainments and alters our concepts of entertainment mode, thereby giving birth to the digital entertainment [7. However, it can take various styles, not limited to film, game, music, theme parks and other established forms of entertainment industries. Entertainment includes "fun" in our everyday life activities, from meeting friends to relaxing at hot spas [8].

Entertainment as an end-product is amusing; as a tool it is powerful. With powerful functionality, it is being applied to all aspects of life from learning, training, designing, communicating and collaborating everywhere. One of the main explorations will be to examine how new forms of mediated interaction can lead to radical new forms of technology and art for entertainment computing 9 .

\subsection{Social and Cultural Dimensions}

All forms of entertainment are having levels of interactivity added to them [10]. An important feature of entertainment is the ability to socialize, by extending the concepts of social networking with novel interactive entertainment concepts. Sociability allows people to assemble based on common interest and personal affinities, creating alternate forms of community.

On the other hand, the arrival of new media strongly influences the forms and content of entertainment. Although it decreases the gap of entertainment styles between people in different areas, at the same time, it causes the losing of local features of traditional cultures, the internal core of various entertainments for different cultures. The entertainment realm keeps on evolving rapidly, but there is still something that keeps constant and cherished by most human beings: 
the culture. Culture is the product of ongoing symbolic human activities that is old and pervasive as human society itself. Entertainment, by contrast, is now primarily connected with the various forms of digital visual pleasures provided by the mass media technology. Bringing culture and entertainment together would make the entertainment experience more unique, meaningful and evoking.

From this point, entertainment can be sustained by the unique aspects of the culture contexts, and makes the experience more meaningful and long-lasting. The cultural background largely depends how we interpret the interactions with others, especially the non-verbal interactions. This cultural dependency allows for a much richer experience to be rendered. This is thanks to the complexity and depth of the semantics involved and the user familiarity with them. There is also the advantage of higher bandwidth of information at the interfaces as symbolic meanings and implicit knowledge can be used. The interface is not limited to explicit messages and meanings anymore. The cultural contexts within entertainment provide spiritual pleasure for human beings 11. Cultural entertainment as the occasions for pleasure is therefore dependent on the shift from sensations to emotions.

Considering contemporary society's fascination with entertainment, we think it is very necessary to explore enriched entertainment styles from everyday life, which highlight the social and cultural grounding of interactive entertainment.

\subsection{Food: A Significant Facilitator of Entertainment}

There are various reasons why food can be a promising medium to explore in the social and cultural contexts of entertainment. Except for providing basic nutrients, food is also considered as a symbolic medium with internal emotional attachments to express and trigger positive feelings. Food can alleviate depression and provide comfort, and eating in itself can serve as a very relaxing, stressrelieving activity. Eating and drinking are modes of entertainment for human beings; humans get pleasure out of gustatory information obtained from food.

Furthermore, we believe food is one of best and most enjoyable social networking platforms - individuals interact through and around it. Food is the glue that bonds people connected to their family, friends and neighbourhoods on the occasions like family dinner, parties, and get-together. Pleasure and family connectedness are included among the several positive aspects of people's interaction with food [5]. Food has also been used as a metaphor to represent culture and identity, the nostalgic longing and consumption of particular food items sustain one's sense of cultural, familial and self-identity. Most of us would admit that, "One way of exploring a culture is through their food and cultural food practices. For example, serving food is considered as typical and representative of the politeness and hospitality in Asia. These viewpoints verify the potential of augmenting food as a novel but intuitive medium to provide enhanced social interaction and playful cultural experience.

Over the years, interaction designers have recently begun to explore the area of human-food-interaction and its implications for design. These explorations have ranged from positive social concepts around human-food interaction and 
how technology can create new kinds of engagements 1213 . Some existing intersections between food and technology mainly take the form of food social network sites, online cooking and farming games, and restaurant recommender or ratings applications. Parallel to this, there have also been some explorations towards playful interaction design on the actual food and food-related activities. "Mamagoto" 14 is an interactive and context-aware dining system which encourages small children to "play" with food, using their curiosity towards food to expand their sensory experience while eating. DinnerWare 15] is an exploration of eating as a medium for computation and aesthetic expression. It consists of a dining service electronically equipped to react to the properties of the food and respond to a user's gestures. Gamelunch [16, a sonically augmented dining table, maps the usual dining actions like cutting and slicing onto physically-based sound synthesis. MetaCookie [17] realized "Pseudo-gustatory" display that represents the desired flavors of cookie by means of a cross-modal effect elicited by visual and olfactory augmented reality. EaTheremin is a fork-type device that enables users to play various sounds by eating foods, which changes the act of eating into practical daily entertainment [18. Playful Tray 19] is embedded with an interactive game play over a weight-sensitive tray surface, and uses children's eating actions as game inputs for reducing their poor eating behaviours.

These works augmented the affordances of actual food by embedding various sensors and actuators over food or tableware. However, they mainly concentrate on the personal human-food interaction, instead of human-to-human interaction mediated by food. Furthermore, none of these studies has looked closely at the manipulation and interaction process around food, and what aspects of this interactive and collaborative process could yield insights into the design of technology and everyday rituals. Different from this, we explore how technology can be designed to shape and enrich the way people interact with food and further enhance the social and cultural food experiences.

Food is not simply a source of nutrition, but is also central to the production of community life, a site for personal and shared reflection and story-telling [20, a medium for social interaction and a symbol of personal identity. Food preparation and sharing in particular provide opportunities to support the creative, sensory, aesthetic and social nature of human-food-human interaction. Furthermore, food related behaviours, such as shared meals, food creation, food preparation and so on, can all be considered to form an integral part of social and personal wellbeing. Could food interaction design also be an important and useful metaphor for the future practice of user experience itself [21]?

Rather than the external playfulness and fantasies, we explore new interaction styles into the wider implications (human, social, cultural, and ethical) of experiences and try to apply it onto food. Extending the growing literature in this area, we look to expand the range of food experience by examining the in-depth roles of food in supporting positive physical and emotional responses, with a focus on the social and situated nature of food interactions, the internal physicality, sociability and cultural recollection. 


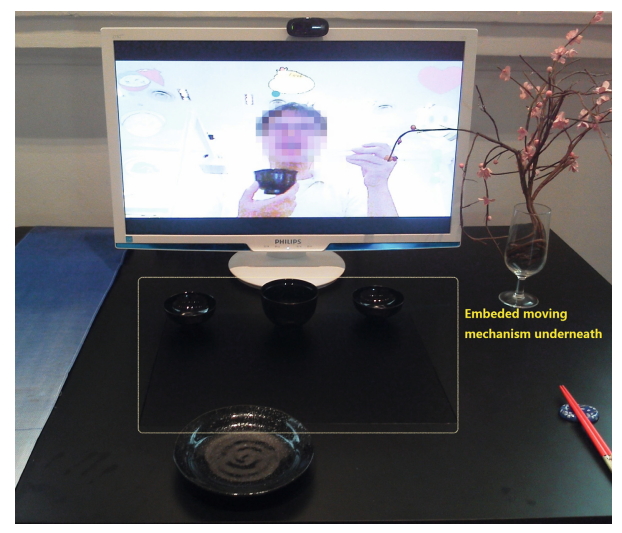

Fig. 1. Overview of the ServeDish Table in dining scenario

\section{Prototypes towards "Leisure Food"}

The domain of entertainment is much broader than just games and leisure activities; entertainment can also take forms in our everyday activities in an interactive and playful way, and designing for this kind of playfulness involves creating objects that elicit a playful approach and provide enjoyable experiences [22]. To design for meaningful and positive interactions, it is essential to identify daily food practices and the opportunities for the design of technology to support such practice. To do this, we need to fully understand the role of technologies to support human-food interactions in social settings.

Towards the "Leisure Food" paradigm, we rely on the cultural understanding of human activities to create expressive interactions and entertainment. The various ways that people find pleasure in food can all serve as springboards for technological designs that reflect or augment the ways to provide new hedonic food experiences for people. In general, food consumption involves many sensory and aesthetic experiences such as cooking meals, sharing and eating food. In this section, we present two prototypes that draw from people's routine activities with food, encourage them with interactive technologies and extend them to remote situations to achieve the shared entertainment experience.

\subsection{ServeDish Table: Enlighten Eating Culture}

Eating food together is always compelling to people, it creates a warm atmosphere that keeps people happy, occupied and entertained. Considering one of the positive aspects about food interaction while eating together, we developed ServeDish Table to mimic the mutual food serving activity in dining situation to provide the warm feelings of pleasure and intimacy, even if people are physically apart. In many Asian cultures, serving food is a conventional dining etiquette to show respect, love and care to family members and guests as well. This tradition has been deemed as a kind of spontaneous activity in our culture, something 


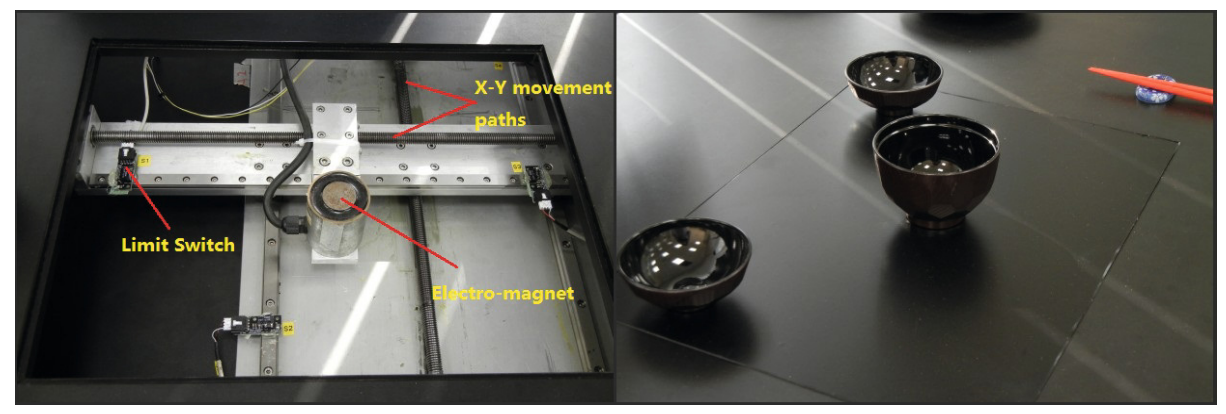

Fig. 2. (Left)Mechanism of ServeDish Table (Right)Actual snapshot when serving dish remotely

that we regularly and unconsciously engage in. Using this system, we attempt to bring back this ritual dinner etiquette and derive emotional pleasure from this physical interaction via food and tableware, even when people fail to share the same dining table.

This prototype includes the visual interface part and the execution mechanism. The visual interface can be a TV monitor embedded with a video camera and Kinect Sensor [23] to track the user's hand movement and achieves gesturebased on-screen menu selection. This function allows the user on one side to easily select one dish, and then the signal would be transmitted to the remote location, triggering the corresponding mechanism to execute the food serving on the other side. Figure 1 is an overview of this system in the dining scenario.

To achieve the remote food serving, we designed the execution mechanism and embedded it into the normal dining table. The mechanism applies the basic principle of magnetic attraction, combined with two-axis linear movement structure, as shown in figure 2 (Left). Permanent magnets are attached on the bottom of the tableware, including bowls and plates. By controlling the movement of electro-magnet component underneath, we can control the movement of the dishes on the table. We chose to use magnets because they are simple to implement, easy to control, and require no wires. When the master controller receives the activation signal, the motors and magnets are activated accordingly to execute the remote moving of dishes on the table surface. Figure 2 (Right) is a screenshot of the actual food serving when user selects one dish.

Using two connected systems in different locations, people can initiate the remote mechanism through gesture interaction with the visual interface to choose one dish to serve, thus to carry out the remote interaction in a physical way. When user on the other side selects one of the dish icons from the screen, let's say, serving rice dish on their partner's table, the electro-magnet will move directly to the position of that dish, activate the magnet, and drag the rice smoothly towards the user on the table surface.

Although different from the actual food serving face-to-face, this simulated activity on physical dish during eating provides a new channel for playful interaction using food. Furthermore, we believe the actual movement of dishes can 
create a feeling of "magic moving", surprising and delighting the receiver, and evoke increased cheering and comforting emotions. Through rethinking how we approach everyday food behaviours, we propose this design of interactive system to engage individuals' socio-cultural reflection on food practices. Although it is not like the common amusement we get from games or movies, the interactivity and cultural retrospective can convey more delicate human emotions and stronger feeling of warmth, which comes in accordance with our theme of "Leisure Food".

\subsection{Food Messaging: Playful and Customized Cooking for Expression}

In addition for eating, food as gifts are also culturally appropriated and encouraged, especially during festivals, anniversaries and celebrations, for example, sending the specially-designed food with personal messages. Cooking and sharing food are important activities to enhance the relationship and intimacy, transmitting warm feelings among people. We would all agree that eating is a form of entertainment experience, so as the cooking process. What about cooking or eating the customized food with special messages inside? As a reproduction and extension of the social fun around food creation and gifting, we developed this food messaging system, which transformed the normal messaging process into a new channel for meaningful and emotional expressions using organic food.

Although it is now almost effortless to send Short Message Service (SMS), digital photos or gift, these intangible messages miss out the physical and emotional sense of care. Instead, imagine sending an edible "Hello" to your remote family members by displaying it on a cake in their kitchen. Food Messaging achieves this by reproducing the digital greetings (i.e. Messages and gift) using edible food materials, which makes tangible the digital messages, creating personalized expressions and gifting in edible format.

To allow the real-time design, transmission, and crafting of self-designed pattern, the system consists of the application software on the mobile side and the food crafting mechanism. User's finger movement is tracked in real time, and the detected pattern is sent to the mechanism via the server in between, then the synchronous crafting is done in three dimensions with multiple flavors using accurate step-motor control and triple-syringed food material injection, through a layer-by-layer printing process. The crafting process, including the motor movements and food materials change, is controlled precisely in accordance with the user's input design. Figure 3 gives an overview of the system structure.

The input interface is designed as an easy-to-use mobile application that allows users to create their preferred food patterns virtually. User can compose their unique messages or patterns by scrawling and writing on the touch screen. They can also change the shapes, colors, text, and even taste of the messages, by tapping on different food materials during the design. Accordingly, the system is designed to craft with multiple food materials, the purpose is not only to make the food messages colorful visually and more tasty, but also enable the creation of contextual messages with changed color, smell and flavor, to represent 


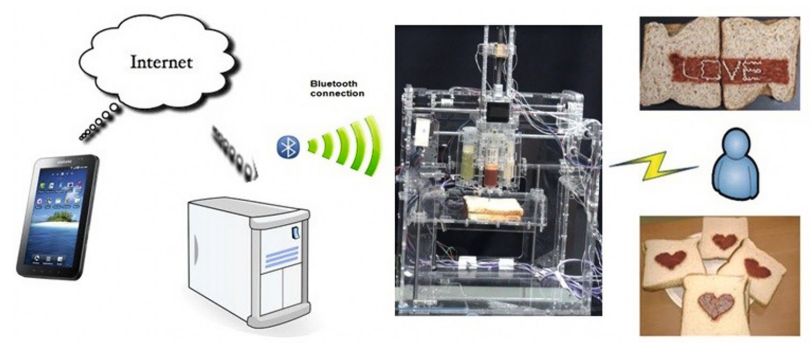

Fig. 3. System design of the Food Messaging prototype

different emotions or feelings. User can also compose different patterns for different layers, thus to construct the 3 -dimensional food with changed taste in each layer. When people are drawing on the screen, their fingers' movements are tracked, and then the detected design is divided into a series of segments, each tagged with their direction and length. When user pressed the "print" button, all the data would be transmitted to the master board of the food crafting mechanism, to indicate the movement route in each axis, thus to exert the crafting process accurately.

The food crafting mechanism functions as the output of the user's input design. Controlled by the master board, this mechanism is supposed to craft the corresponding pattern received from the design interface with real edible food. For accurate food crafting, we employ the motor-controlled syringe injection on a 3-axis robotic carriage. Basically, the model is designed to use 3-degree freedom robotic carriage to move the food depositing component along the $\mathrm{x}$ and $\mathrm{y}$ axis, and the platform up and down to form the $\mathrm{z}$ coordinate of the system. The accurate and smooth movement of each axis is achieved through lead-screw coupling powered by step motor, controlled by the microchip from the master board. Through balancing the coordinate movement of syringe position and food extrusion, the mechanism is able to form any designed shape using different food materials.

When receiving the interpreted data of user's design, the step motors would start accordingly to move the robotic carriage. Simultaneously, the robotic carriage moves the attached food depositing component along the $\mathrm{x}$ and $\mathrm{y}$ axis to complete one layer. When finishing the top layer, the platform will move down a certain distance to continue with the next layer. Material change is achieved by rotation of depositing syringe to a certain degree. Through this layer-by-layer crafting, a piece of 3-dimensional physical food can be constructed. Using this system, people can creatively compose their message-embedded food, and transmit it to remote loved ones as a unique gift, showing greetings and care in a physical format. It is an innovative way of customized cooking and social interaction through food, also conveying love and care physically to remote people.

Messaging and gifting through food demonstrates a subtlety and courtesy that messaging through digital devices sometimes lacks. Compared with plain 


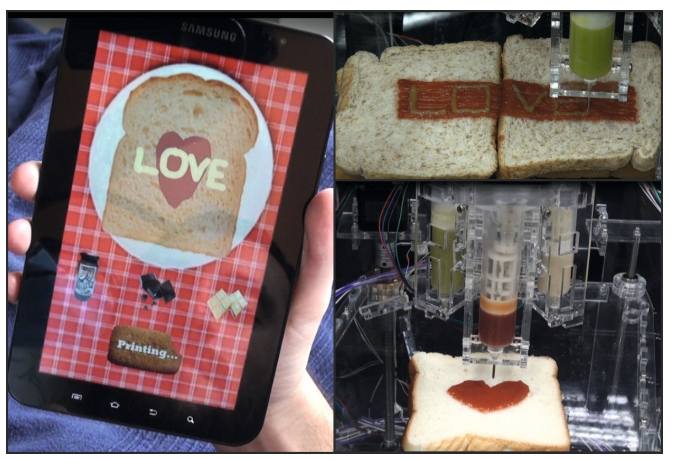

Fig. 4. Examples from interaction with the Food Messaging prototype

text, or digital message like SMS or twitter, expression through organic food can convey a stronger sense of emotional warmth and connectedness. It is more than simply saying "I love you" or "I miss you", it is a physical embodiment of care and affection of one person to others [24], not only visually, but also with smell and taste. Food here is the multisensory medium for remote communication and entertainment, and each flavor can be translated into a corresponding emotional state or special experience from one's memory. This flavor-changed eating experience of food acts as a meaningful way for emotional expression.

This prototype provides an alternative approach of engaging with food. By sending routine, social touch messages, such as birthday greetings, good luck messages and other general expressions of affection, people actually involve themselves into the creative and personalized cooking process. The richness of entertainment experience can be much increased and intensified by this playful and interactive way of personalized food creation and expression. Rather than focusing on the information transmission, it emphasizes more on the enriched food experience, which engages people into the social communication and entertainment over food, in a playful and meaningful way. It enhances the engagement to connect digital playfulness to active participation in the target activity of cooking and eating.

\section{Evaluation and Discussion}

To investigate the actual effectiveness of the working prototypes, we conducted the preliminary user study to see whether they reach the intention of proposed "Leisure Food" theme, and get a deeper understanding of the user perception.

We recruited 29 volunteers (17 male and 12 female) from the general public, aged from 20 to 40 years old. They are from different countries, like China, India, Japan, Sri Lanka, Korea, and Spain also. After explaining the general concept of the two systems and how to use them, we asked every user to use each system for about 5 minutes (We did not apply the remote setting, so that user can 
activate the system and see the output at the same location). Then we use the questionnaire to elicit their responses about what they think and feel about the systems, in terms of level of engagement and evoked cultural recalling. The participants were given the questionnaire right after their usage both systems.

Regarding the questionnaire, we use the Likert Scales [25], a series of statement to which the respondents rate their level of agreement on a 5-point scale. The rankings from 1 to 5 indicate from "Strongly Disagree" to "Strongly Agree". In this study, we focused on the two attributes for the users to evaluate: 1 . The system increases the engagement experience; 2 . The system supports the cultural recollection experience. The participants were required to rate their agreement on these two statements regarding each prototype.

What's more, to avoid the "social desirability bias" [25, we collect the posttest data in a way that the evaluator did not see the responses until the participant has left, and we also made the survey itself anonymous to elicit more honest reactions. Data collected in the survey are expressed by histogram in percentage.

Regarding the engagement experience of the ServeDish Table, we got very positive ratings from the users. Participants reported a mean value of 4.11 $(\mathrm{SD}=0.557)$, indicating the level between "Agree" and "Strongly Agree". As shown in figure 5 (Left), a great majority of participants admitted that the interaction of serving dish remotely can enhance their engagement experience while eating, except for $10 \%$ of people who rated neutral on this statement.

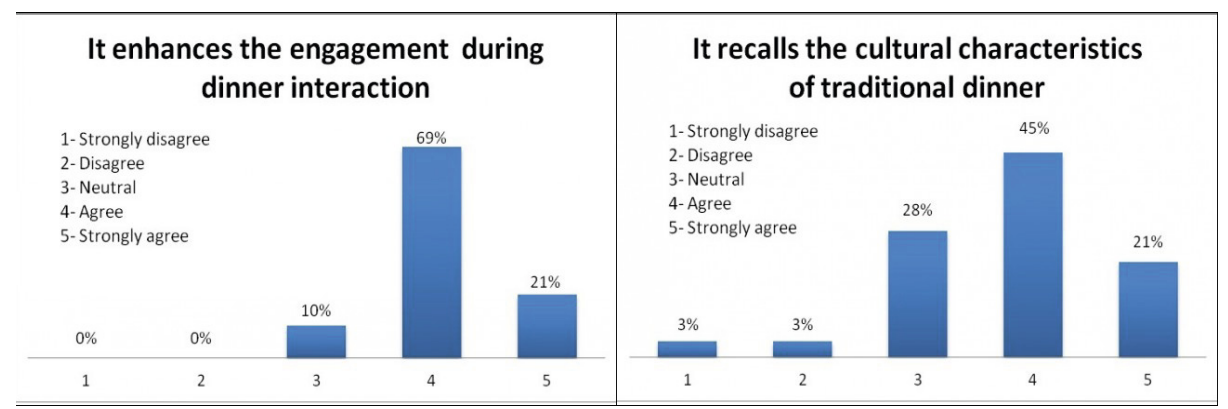

Fig. 5. Users' feedback regarding the interaction experience with ServeDish Table

The ratings on the cultural experience through this prototype are slightly different from the engagement aspect. Participants reported a mean value of 3.76 $(\mathrm{SD}=0.951)$, somewhere between neutral and agree. The responses are generally lower and more varied on this attribute than the engagement aspect, probably because people from different backgrounds have different awareness and perception levels regarding this dining culture. People grown up in western lifestyle (e.g. Spain) may have no idea about this etiquette and feel less engaged, so it would be more difficult for them to understand the underlying grounding in culture that reflected from the physical manipulation of dishes from another person. 
As for the engagement level triggered by the Food Messaging system, participants reported an average value of 4.11, similar with the ServeDish Table but with larger variance $(\mathrm{SD}=0.860)$, having more people rated "Strongly Agree" for this prototype (figure 6). From this, we can tell that participants would be more motivated and delighted to be creative in the sending of playful and inventive messages through this system than would be the case if they were sending content to a mobile phone. On the other hand, the level of culture recollection was rated lower. They reported a mean value of $3.448(\mathrm{SD}=0.948)$ regarding the cultural experience, $42 \%$ rated as "Agree" and 10\% rated "Strongly Agree".

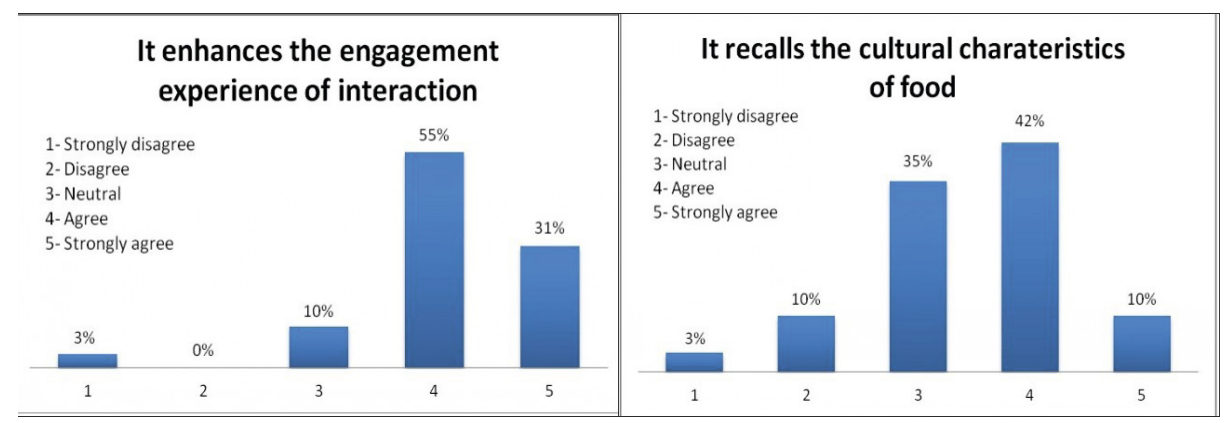

Fig. 6. Users' feedback regarding the interaction experience with Food Messaging

This preliminary study provides a snapshot into the users' perception of the two systems. Users' overall reactions were quite positive. They found the prototypes really provide more enjoyment for food cooking and eating, and they were a pleasure to use. As one user reported, "I am really surprised when the bowl begins moving towards me, and it makes me feel much more excited when eating". Similarly, another user mentioned, "It was really great to see my drawings actually been printed out on a piece of bread, and I am sure my Mum would be happy to receive it." However, they also expressed some concerns about the prototypes, for example, the set up of the system, the noise from the motors, and cleaning issues, all of which are of great help for the further improvements.

This evaluation has demonstrated that both of these two prototypes achieve the increased entertainment experience with cultural recollection in a certain degree. In comparison, users gave higher ratings on the engagement aspect than the cultural feeling experience regarding both prototypes. Although these two prototypes basically achieve the two proposed affordances of "Leisure Food", this slight difference indicated us the necessity to explore further into the grounding culture of food interaction, so as to gain more insights on how to reflect and highlight the culture embodiment through these food interactions.

Towards enriched hedonic experience of food interaction, we need to understand more about the positive aspects of the human experience with food. It is at this point that we can begin to design for "Leisure Food"; using technologies to reconstruct and augment the usual way that people interact with foods. 


\section{Conclusion}

Entertainment is now a key driver in the development of technology. In this paper, we leverage on the rich potentials of food and propose the notion of "Leisure Food", as a paradigm to empower food with technology towards enhanced social and cultural entertainment. "Leisure Food" aims to expand the human-food interaction and create technologies or applications that embrace the positive, and delightful aspects of food practices as social and cultural experience.

Whether noticed or not, the practice around food is actually about food and also what people consider as significant in the process of cooking and eating food. This notion relies on people's customary social manipulations and culturally responsive activity with food, and then extends them to remote situations to achieve enriched shared entertainment. We describe how studying the positive aspects of people's interactions with food can lead to designing very different types of entertainment technologies. The two examples in this paper are some of the ways that we can think about the positive ways that people interact with each other through food, the food sharing and serving while eating together, and the creativity expression and symbolic gifting through cooking.

As we move on to explore and create "Leisure Food", it is important to draw from and embrace the deep and wide cultural repository of human experiences with food, discover the significant values human have towards food and then take a closer look at how to harness such values to enhance the enjoyable aspects of food entertainment. Humanity possesses a rich pool of cultural experiences which can contribute fundamental resources to the embodied interaction design [26], and to the entertainment as well. It is necessary to develop a better understanding about how to go beyond the amusement from mass media and into encouraging positive experience. We hope the future research can explore further to design embodied interaction based on cultural practice, thus to derive social and cultural entertainment out of human's internal values and identities.

Acknowledgments. We thank our colleagues from Keio-NUS CUTE Center of National University of Singapore, who helped in the building of the prototypes.

\section{References}

1. Stromberg, P.: Person and Community in the Culture of Entertainment. Pastoral Psychology, 1-8 (2010)

2. Cheok, A.D., et al.: Social and physical interactive paradigms for mixed-reality entertainment. Comput. Entertain. 4(2), 5 (2006)

3. Alben, L.: Quality of experience: defining the criteria for effective interaction design. Interactions 3(3), 11-15 (1996)

4. Finkelstein, J.: The Taste of Boredom: McDonaldization and Australian Food Culture. American Behavioral Scientist 47(2), 187-200 (2003)

5. Grimes, A., Harper, R.: Celebratory technology: new directions for food research in HCI. In: Proceeding of CHI 2008, pp. 467-476. ACM, Florence (2008)

6. Quandt, S.A., et al.: The social and nutritional meaning of food sharing among older rural adults. Journal of Aging Studies 15(2), 145-162 (2001) 
7. Jin, Z.: Global Technological Change: From Hard Technology to Soft Technology - Second Edition. Intellect Ltd. (2011)

8. Shedroff, N.: Experience Design 1. New Riders Press, New Riders (2001)

9. Cheok, A.D.: Art and Technology of Entertainment Computing and Communication. In: Advances in Interactive New Media for Entertainment Computing, pp. 1-18. Springer (2010)

10. Lampel, J., Shamsie, J., Lant, T.K.: The business of culture: strategic perspectives on entertainment and media. Lawrence Erlbaum Assoicates (2006)

11. Salem, B., Rauterberg, M., Nakatsu, R.: Kansei Mediated Entertainment. In: Harper, R., Rauterberg, M., Combetto, M. (eds.) ICEC 2006. LNCS, vol. 4161, pp. 103-116. Springer, Heidelberg (2006)

12. Hirsch, T., et al.: Making food, producing sustainability. In: Proceedings of the CHI 2010 Extended Abstracts, pp. 3147-3150. ACM, Atlanta (2010)

13. Bell, G., Kaye, J.: Designing Technology for Domestic Spaces: A Kitchen Manifesto. Gastronomica 2(2), 46-62 (2002)

14. Arakawa, T., Inakage, M.: Mamagoto: "playing" with food. In: ACM SIGGRAPH 2007 Posters, p. 161. ACM, San Diego (2007)

15. Coelho, M.: DinnerWare: why playing with food should be encouraged. In: Proceedings of the CHI 2009 Extended Abstracts on Human Factors in Computing Systems, pp. 3505-3506. ACM, Boston (2009)

16. Polotti, P., et al.: Gamelunch: forging a dining experience through sound. In: CHI 2008 Extended Abstracts on Human Factors in Computing Systems, pp. 22812286. ACM, Florence (2008)

17. Narumi, T., Nishizaka, S., Kajinami, T., Tanikawa, T., Hirose, M.: Augmented reality flavors: gustatory display based on edible marker and cross-modal interaction. In: Proceedings of CHI 2011, pp. 93-102 (2011)

18. Kadomura, Nakamori, R., Tsukada, K., Siio, I.: EaTheremin. In: SIGGRAPH Asia 2011 Emerging Technologies on - SA 2011, p. 1. ACM Press (2011)

19. Lo, J.-L., Lin, T.-y., Chu, H.-h., Chou, H.-C., Chen, J.-h., Hsu, J.Y.-j., Huang, P.: Playful Tray: Adopting Ubicomp and Persuasive Techniques into Play-Based Occupational Therapy for Reducing Poor Eating Behavior in Young Children. In: Krumm, J., Abowd, G.D., Seneviratne, A., Strang, T., et al. (eds.) UbiComp 2007. LNCS, vol. 4717, pp. 38-55. Springer, Heidelberg (2007)

20. Bh, M.T., et al.: 4Photos: a collaborative photo sharing experience. In: Proceedings of the 6th Nordic Conference on Human-Computer Interaction: Extending Boundaries, pp. 52-61. ACM, Reykjavik (2010)

21. Venkatacharya, P.S., et al.: What can user experience learn from food design? In: Proceedings of the 27th International Conference Extended Abstracts on Human Factors in Computing Systems, pp. 3287-3292. ACM, Boston (2009)

22. Lucero, A., Arrasvuori, J.: PLEX Cards: a source of inspiration when designing for playfulness. In: Proceedings of the 3rd International Conference on Fun and Games, pp. 28-37. ACM, Leuven (2010)

23. Microsoft Xbox Kinect, http://www.xbox.com/kinect

24. Perry, M., Rachovides, D.: Entertaining Situated Messaging at Home. Comput. Supported Coop. Work 16(1-2), 99-128 (2007)

25. Tullis, T., Albert, B.: Chapter 6 - Self-Reported Metrics. In: Measuring the User Experience, pp. 123-166. Morgan Kaufmann, San Francisco (2008)

26. Lindtner, S., Anderson, K., Dourish, P.: Cultural appropriation: information technologies as sites of transnational imagination. In: Proceedings of CSCW 2012, pp. 77-86. ACM (2012) 